\title{
Disseminated intravascular coagulation in catastrophic antiphospholipid syndrome: clinical and haematological characteristics of 23 patients
}

\author{
R A Asherson, G Espinosa, R Cervera, J A Gómez-Puerta, J Musuruana, S Bucciarelli, M Ramos- \\ Casals, A L Martínez-González, M Ingelmo, J C Reverter, J Font, D A Triplett, for the Catastrophic \\ Antiphospholipid Syndrome Registry Project Group*
}

\begin{abstract}
Background: Disseminated intravascular coagulation (DIC) is an acquired syndrome characterised by formation of microthombi and fibrin deposition in the microvasculature. The catastrophic antiphospholipid syndrome (APS) is characterised by multiorgan thrombosis, mainly involving small vessels. A broad spectrum of disorders may develop DIC features; however, the catastrophic APS has not previously been recognised as a cause of DIC.

Objective: To analyse the clinical and laboratory characteristics of catastrophic APS patients with DIC features.

Methods: The web site based international registry of patients with catastrophic APS (CAPS registry) (http:// www.med.ub.es/MIMMUN/FORUM/CAPS.HTM) was analysed and the cases with DIC features selected.

Results: In 173 patients with catastrophic APS, 23 (13\%) were found with DIC features. The clinical and immunological characteristics were similar in catastrophic APS patients with and without DIC features; a significant difference was found only in the prevalence of thrombocytopenia (100\% in patients with DIC features $v 59 \%$ in those without DIC features).

Conclusions: DIC features are not rare in catastrophic APS, supporting the need for systematic screening of antiphospholipid antibodies in all patients with DIC features without precipitating factors. The presence of DIC features in the context of an APS makes it imperative to rule out the catastrophic variant of this syndrome.
\end{abstract}

D isseminated intravascular coagulation (DIC) is an acquired syndrome characterised by the widespread activation of coagulation with occlusion of small and medium sized vessels. This condition may compromise the blood supply to organs and contribute to multiorgan failure. It is not a disease entity in itself but always occurs as a complication of an underlying disorder, the most common being infection, severe trauma, malignancy, and obstetric complications. ${ }^{1-3}$

The "catastrophic" variant of the antiphospholipid syndrome (APS) is an accelerated form of this syndrome resulting in multiorgan failure because of multiple small vessel occlusions. ${ }^{4}$ As with DIC, most of the catastrophic APS episodes are preceded by a precipitating event, such as infection, surgery or trauma, obstetric complications, and malignancies. ${ }^{56}$ Furthermore, laboratory features of DIC were reported in $19-28 \%$ of the largest catastrophic APS series. ${ }^{78}$

Our objective in the present study was to analyse the clinical and laboratory characteristics of catastrophic APS patients with DIC features to determine whether these patients form a special subset within the catastrophic APS population.

\section{METHODS}

We analysed the web site based international registry of patients with catastrophic APS (the CAPS registry; http:// www.med.ub.es/MIMMUN/FORUM/CAPS.HTM). This registry was created in 2000 by the European Forum on Antiphospholipid Antibodies and compiles all the published reports as well as newly diagnosed cases from all over the world. Up to October 2003 it included 220 patients with this condition. ${ }^{9}$ We selected those patients who had some of the laboratory features of DIC (raised fibrin related markers, decreased fibrinogen concentrations, or both). Isolated thrombocytopenia and prolonged prothrombin time were not considered to be selection criteria as they can be manifestations of the APS.

According to the International Scientific Subcommittee for DIC, we calculated the DIC score as follows:

- platelets: $>100 \times 10^{9} / \mathrm{l}=0 ;<100 \times 10^{9} / \mathrm{l}=1 ;<50 \times 10^{9} / \mathrm{l}$ $=2$;

- raised fibrin related markers (fibrin degradation products and D-dimers): no increase $=0$; moderate increase $=2$; marked increase $=3$;

- prolonged prothrombin time: $<3^{\prime}$ seconds $=0,3-6$ seconds $=1 ;>6$ seconds $=2$;

- decreased fibrinogen: $>1.0 \mathrm{~g} / \mathrm{l}=0 ;<1.0 \mathrm{~g} / \mathrm{l}=1$.

Overt DIC was diagnosed when the total score was $\geqslant 5$; a score $<5$ was considered suggestive of DIC. ${ }^{10}$

Fisher's exact test (bilateral) was employed for the statistical analysis, using the SPSS 10.0 statistical program.

\section{RESULTS}

\section{General characteristics}

Of the 220 patients included in the CAPS registry, information on DIC features was not available in 34 cases and there were incomplete data for DIC scoring in 10 further patients. This left a total of 176 patients available for analysis. Of these, $23(13 \%)$ had DIC features associated with catastrophic APS: $17(74 \%)$ female and six (30\%) male, mean (SD) age 39 (13) years (range 11 to 60 ). Ten $(43 \%)$ suffered from primary APS, nine (39\%) had systemic lupus erythematosus (SLE), three $(13 \%)$ had lupus-like disease, and one $(4 \%)$ had polychondritis.

Abbreviations: aCL, anticardiolipin antibodies; APS, antiphospholipid syndrome; DIC, disseminated intravascular coagulation 
Table 1 Laboratory variables according the scoring system for DIC

\begin{tabular}{|c|c|c|c|c|c|c|c|c|c|c|}
\hline Case & $\begin{array}{l}\text { Platelets } \\
\left(\times 10^{9} / \mathrm{I}\right)\end{array}$ & Score & FDPs & D-dimers & Score & PT (s) & Score & Fibrinogen (g/l) & Score & Total \\
\hline 1 & 128 & 0 & Increased & NR & 3 & 13.6 & 0 & 4.98 & 0 & 3 \\
\hline 2 & 57 & 1 & Increased & NR & 3 & 12.7 & 0 & 6.5 & 0 & 4 \\
\hline 3 & 39 & 2 & NR & Increased & 3 & NR & NA & 1.27 & 0 & 5 \\
\hline 4 & 21 & 2 & Increased & Increased & 2 & NR & NA & Low & 1 & 5 \\
\hline 5 & 67 & 1 & Increased & NR & 2 & NR & NA & 0.84 & 1 & 4 \\
\hline 6 & 80 & 1 & Increased & NR & 3 & 32 & 2 & 0.7 & 1 & 7 \\
\hline 7 & 47 & 2 & Increased & NR & 2 & NR & NA & 4.0 & 0 & 4 \\
\hline 8 & 50 & 2 & Increased & NR & 3 & NR & NA & 1.8 & 0 & 5 \\
\hline 9 & "Low" & NA & NR & Increased & 2 & NR & NA & Low & 1 & 3 \\
\hline 10 & 12 & 2 & Increased & NR & 2 & 19 & 2 & 6.49 & 0 & 6 \\
\hline 11 & 35 & 2 & Increased & NR & 2 & 10.4 & 0 & 0.0024 & 1 & 5 \\
\hline 12 & 57 & 1 & Increased & NR & 3 & 42 & 2 & NR & NA & 6 \\
\hline 13 & 34 & 2 & Increased & Increased & 3 & 16.7 & 2 & 4.6 & 0 & 7 \\
\hline 14 & 16 & 2 & Increased & NR & 2 & Prolonged & 1 & NR & NA & 5 \\
\hline 15 & 16 & 2 & Increased & Normal & 2 & NR & NA & NR & NA & 4 \\
\hline 16 & 86 & 1 & Increased & NR & 2 & NR & NA & NR & NA & 3 \\
\hline 17 & 31 & 2 & NR & Increased & 2 & Prolonged & 1 & 4.65 & 0 & 5 \\
\hline 18 & 127 & 0 & Increased & Increased & 3 & NR & NA & 3.12 & 0 & 3 \\
\hline 19 & 77 & 1 & Increased & NR & 2 & NR & NA & NR & NA & 3 \\
\hline 20 & 61 & 1 & Increased & NR & 3 & NR & NA & NR & NA & 4 \\
\hline 21 & 16 & 2 & Increased & NR & 3 & NR & NA & 5.51 & 0 & 5 \\
\hline 22 & 70 & 1 & Increased & Increased & 2 & NR & NA & NR & NA & 3 \\
\hline 23 & 35 & 2 & NR & Increased & 2 & Normal & 0 & NR & NA & 4 \\
\hline
\end{tabular}

Platelet count: $>100 \times 10^{9} / \mathrm{I}=0 ;<100 \times 10^{9} / \mathrm{I}=1 ;<50 \times 10^{9} / \mathrm{I}=2$.

Raised fibrin related markers (FDPs and D-dimers): no increase $=0$; moderate increase (raised but less than twice the normal level) $=2$; marked increase (more than twice the normal level) $=3$.

Prothrombin time: $<3$ seconds $=0 ; 3-6$ seconds $=1 ;>6$ seconds $=2$.

Fibrinogen concentration: $>1.0 \mathrm{~g} / \mathrm{l}=0 ;<1.0 \mathrm{~g} / \mathrm{l}=1$.

Total score: $\geqslant 5$, compatible with $\mathrm{DIC} ;<5$, suggestive of DIC.

DIC, disseminated intravascular coagulation; FDPs, fibrinogen degradation products; NA, not available; NR, not recorded; PT, prothrombin time.

\section{Clinical presentation and precipitating factors}

Intra-abdominal involvement was identified in all 23 patients, mainly consisting of renal $(78 \%)$, hepatic $(48 \%)$, gastrointestinal $(39 \%)$, splenic $(17 \%)$, pancreatic $(9 \%)$, and adrenal (9\%) manifestations.

Pulmonary complications were reported in 16 patients (70\%), mainly acute respiratory distress syndrome (ARDS) and confirmed pulmonary embolism, but occasionally intraalveolar haemorrhage. Eleven patients (48\%) had cardiac involvement, mainly cardiac failure and confirmed myocardial infarction or valve lesions. Fifteen patients (65\%) had evidence of cerebrovascular complications, mainly encephalopathy and cerebrovascular accidents, but occasionally seizures, headache, or silent brain infarcts. Deep venous thrombosis was present in two patients (9\%) and peripheral arterial occlusive disease in one (4\%).

Skin manifestations were also frequent $(78 \%)$ and consisted of livedo reticularis, ulcers, necrotic lesions, digital gangrene, purpura, microthrombosis of small vessels, splinter haemorrhages, and multiple ecchymosis.

Other lesions occasionally encountered were bone marrow necrosis, mononeuritis multiplex, and retinal involvement.

The most common precipitating conditions were infections (seven cases) and surgical procedures (four cases). Other cases were attributed to drug use and anticoagulation withdrawal (one each).

\section{APS related laboratory findings}

Thrombocytopenia (platelet count $<150 \times 10^{9} / 1$ ) was reported in all 23 patients and evidence of haemolytic anaemia in nine $(41 \%)$, accompanied by schistocytes in five $(23 \%)$. The IgG isotype of anticardiolipin antibodies (aCL) was detected in 19 patients $(83 \%), \operatorname{IgM}$ aCL in eight $(38 \%)$, and lupus anticoagulant in $18(82 \%)$.

\section{DIC features}

A platelet count of $<100 \times 10^{9} / 1$ was reported in 20 patients $(87 \%)$ (in one additional case, the count was reported only non-specifically, as "low platelet count"). Increased fibrin degradation products were reported in all 19 patients in whom they were recorded, and positivity for D-dimers in eight of nine cases (89\%). A prolonged prothrombin time was reported in six of 10 patients $(60 \%)$ and decreased fibrinogen levels were present in five of 13 cases (39\%) (in two cases they were reported non-specifically as "low levels"). Table 1 shows in detail each case of catastrophic APS with DIC features. Eleven patients (48\%) had a DIC score of 5 or above (compatible with overt DIC). The remaining 12 patients (52\%) had a DIC score of 3 or 4 (suggestive of DIC).

\section{Treatment and outcome}

Most patients received a combination of treatments. Anticoagulation were used in 20 patients (87\%), steroids in $19(83 \%)$, plasma exchange in nine $(39 \%)$, cyclophosphamide in eight $(35 \%)$, intravenous gamma globulin in five $(22 \%)$, and splenectomy in two (9\%). Other treatments used were prostacyclin and antithrombin concentrate (one case each).

Recovery occurred in $61 \%$ of catastrophic APS patients with DIC features and in 58\% of those without DIC features (NS). Assessing the use of single treatments, recovery of DIC patients occurred in $58 \%$ of those treated with anticoagulants $v 67 \%$ of those not treated with anticoagulants (NS); in 58\% of those treated with steroids $v 67 \%$ of those not (NS); in $38 \%$ of those treated with cyclophosphamide $v 71 \%$ of those not (NS); in $50 \%$ of those treated with plasmapheresis $v 64 \%$ of those not (NS); and in $83 \%$ of those treated with intravenous gamma globulin $v 50 \%$ of those not (NS).

\section{Comparison of catastrophic APS patients with and without DIC}

The profiles of demographic characteristics (sex distribution and mean age), clinical features (severe organ involvement), and immunological findings (lupus anticoagulant, IgG aCL, and IgM aCL) were similar. Significant differences were found only in the prevalence of thrombocytopenia (100\% in the DIC group $v 59 \%$ in the catastrophic APS patients without 
Table 2 Differential diagnosis of multiorgan thrombotic disorders

\begin{tabular}{|c|c|c|c|}
\hline & $\begin{array}{l}\text { Catastrophic } \\
\text { APS }\end{array}$ & DIC & TMHA \\
\hline \multicolumn{4}{|l|}{ Haemorrhagic } \\
\hline Anaemia & \pm & \pm & + \\
\hline Schistocytes & \pm & \pm & ++ \\
\hline $\begin{array}{l}\text { Thrombocytopenia } \\
\text { Prolonged prothrombin }\end{array}$ & + & + & +r+ \\
\hline $\begin{array}{l}\text { Pime } \\
\text { Prolonged activated } \\
\text { partial thromboplastin }\end{array}$ & - & + & - \\
\hline $\begin{array}{l}\text { time } \\
\text { Fibrinogen }\end{array}$ & \pm & + & (2. \\
\hline $\begin{array}{l}\text { degradation products } \\
\text { Antiphospholipid }\end{array}$ & \pm & + & - \\
\hline antibodies & & & \\
\hline $\begin{array}{l}\text { Plasma ADAMTS-13 } \\
\text { activity }\end{array}$ & Normal? & $\begin{array}{l}\text { Moderately } \\
\text { reduced }\end{array}$ & $\begin{array}{l}\text { Absent* or } \\
\text { severely reduced }\end{array}$ \\
\hline
\end{tabular}

*In cases of familial thrombocytopenic purpura (TTP), acquired idiopathic TTP, and pregnancy related TTP.

ADAMTS-13, von Willebrand factor cleaving protease; APS, antiphospholipid syndrome; DIC, disseminated intravascular coagulation; TMHA, thrombotic microangiopathic haemolytic anaemia.

DIC, $\mathrm{p}<0.001$ ). Other characteristics typically encountered in other states causing DIC-such as renal failure, skin involvement, or ARDS-were not more frequent in catastrophic APS patients with DIC features than in those without.

\section{DISCUSSION}

We observed laboratory features of DIC in at least 13\% of patients diagnosed as having the catastrophic variant of APS. However, it should be borne in mind that there were incomplete data for DIC scoring in 10 reported cases. Thus, under ideal circumstances where all the data were available, the incidence might turn out to be higher.

The pathophysiology of DIC and catastrophic APS is poorly understood, but the two conditions probably share some pathogenic mechanisms and triggering factors. In DIC, enhanced fibrin formation is caused by tissue factor mediated thrombin generation and simultaneous dysfunction of inhibitory mechanisms, such as the antithrombin system and the protein $\mathrm{C}$ and protein $\mathrm{S}$ system. In addition, fibrin removal is impaired because of fibrinolytic system depression, mainly caused by high circulating levels of plasminogen activator inhibitor type 1 (PAI-1). ${ }^{1-3}$ Conversely, catastrophic APS is associated with endothelial cell activation as a result of antigen-antibody reactions on the surface of endothelial cells or monocytes. ${ }^{11}$ Furthermore, inhibition of both protein $\mathrm{C}$ activation and the function of activated protein $\mathrm{C}$ have been observed in association with APS. ${ }^{12}$ Finally, increased plasma concentrations of PAI-1 characterise the hypofibrinolytic state in APS. ${ }^{13}$

A link between DIC and catastrophic APS can be assumed from the original description of DIC by McKay in $1965 .{ }^{14} \mathrm{He}$ described a 38 year old woman with SLE with some features suggestive of APS, such as chorea, mitral valve disease, and spontaneous abortion. A few days after an elective cholecystectomy she developed a sudden episode of multiorgan failure characterised by fever, severe congestive heart failure, ARDS, renal failure, and somnolence accompanied by features of DIC (low fibrinogen, thrombocytopenia, and a prolonged prothrombin time). Her clinical status deteriorated and she died three weeks after the surgical procedure. Necropsy showed microvascular thrombosis of the heart, adrenal glands, lungs, and bone marrow, in addition to a non-bacterial thrombotic endocarditis, all of these being typical features of catastrophic APS. At that time, however, APS was an unknown entity.

Infections associated with DIC were the most common precipitating factors in catastrophic APS in our series of patients. Molecular "mimicry" has been proposed as one of the major mechanisms responsible for the development of catastrophic APS following infections. ${ }^{15}$ Thus an infectious aetiology for the APS, especially its catastrophic variant, should perhaps be considered more frequently and appropriate antibiotic therapy instituted.

Another aspect to bear in mind is the differential diagnosis between DIC and disorders presenting with thrombotic microangiopathic haemolytic anaemia (TMHA). The clinical picture of DIC, TMHA, and APS may overlap and, if they coexist in the same patient, the diagnosis may be difficult at the time of presentation (table 2).

In conclusion, DIC features are not rare in catastrophic APS. This would support the need for systematic screening of antiphospholipid antibodies in all patients with DIC without precipitating factors. In addition, the presence of DIC in the context of an APS makes it mandatory to rule out the catastrophic variant of this syndrome.

\section{Authors' affiliations}

R A Asherson, Rheumatic Diseases Unit, Department of Medicine, University of Cape Town, Faculty of Health Sciences and Groote Schuur Hospital, Cape Town, South Africa

G Espinosa, R Cervera, J A Gómez-Puerta, J Musuruana, S Bucciarelli, M Ramos-Casals, A L Martínez-González, M Ingelmo, J Font, Department of Autoimmune Diseases, Institut Clínic de Medicina i Dermatologia, Hospital Clínic, Barcelona, Catalonia, Spain J C Reverter, Department of Haemostasis and Haemotherapy, Institut Clínic de Malalties Hemato-Oncològiques, Hospital Clínic, Barcelona D A Triplett, Department of Pathology, Indiana University School of Medicine, Midwest Hemostasis and Thrombosis Laboratories, and Department of Pathology, Ball Memorial Hospital, Muncie, Indiana, USA

*The members of the Catastrophic Antiphospholipid Syndrome Registry Project Group who contributed to the study are listed in the appendix

Correspondence to: Dr Ricard Cervera, Servei de Malalties Autoimmunes, Hospital Clínic, Villarroel 170, 08036-Barcelona, Catalonia, Spain; rcervera@clinic.ub.es

Accepted 9 October 2004

\section{APPENDIX}

\section{THE CATASTROPHIC ANTIPHOSPHOLIPID SYNDROME REGISTRY PROJECT GROUP}

The members of the Catastrophic APS Registry Project Group who contributed to this study are as follows:

M-C Amigo, Rheumatology Department, Instituto Nacional de Cardiología, Ignacio Chavez, Mexico City, Mexico; L BarileFabris, Rheumatology Department, Hospital de Especialidades, Centro Medico la Raza IMSS, Mexico City, Mexico; $J-J$ Boffa, Department of Nephrology, Hôpital Tenon, Paris, France; J Chapman, Neuroimmunology Service, Tel Aviv Sourasky Medical Centre, Tel Aviv, Israel; C Davidson, Department of Cardiology, Royal Sussex Hospital, Brighton, UK; A E Denes, Division of Oncology, Department of Medicine, Washington University School of Medicine, St Louis, Missouri, USA; $R \quad H \quad W \quad M$ Derksen, Department of Rheumatology and Clinical Immunology, University Medical Centre, Utrecht, Netherlands; J F Diaz Coto, Caja Costarricense del Seguro Social, San Jose, Costa Rica; $P$ Disdier, Service de Medecine Interne, Centre Hospitalier Universitaire Timone, Marseille, France; $R$ Egan, Department of Medicine, University of Kentucky Medical Center, Lexington, Kentucky, USA; $M$ Ehrenfeld, Chaim Sheba Medical Centre and Tel-Aviv University, Tel-Hashomer, 
Israel; $R$ Enriquez, Nephrology Section, Hospital General de Elche, Spain; F Falcini, Department of Paediatrics, University of Florence, Italy; L S Fang, Renal Associates, Massachusetts General Hospital and Harvard Medical School, Boston, Massachusetts, USA; J T Grandone, Neenah, Wisconsin, USA; A Gurjal, Division of Hematology/Oncology, Barbara Ann Karmanos Cancer Institute, Detroit, Michigan, USA; G Hayem, Department of Rheumatology, CHU Bichat-ClaudeBernard, Paris, France; $G R V$ Hughes, Lupus Research Unit, The Rayne Institute, St Thomas' Hospital, London, UK; $S$ Inam, Riyadh Armed Forces Hospital Riyadh, Saudi Arabia; $K$ Shashi Kant, Department of Internal Medicine, University of Cincinnati College of Medicine, Ohio, USA; $M$ A Khamashta, Lupus Research Unit, The Rayne Institute, St Thomas' Hospital, London, UK; C S Kitchens, Department of Medicine, University of Florida, Gainesville, Florida, USA; M J Kupferminc, Department of Obstetrics and Gynaecology, Lis Maternity Hospital, Tel Aviv University, Tel Aviv, Israel; $R$ A Levy, Department of Rheumatology, Faculdade de Ciencias Medicas, Universidade do Estado do Rio de Janeiro, Rio de Janeiro, Brazil; S F Lui, Department of Medicine, Prince of Wales Hospital and Chinese University of Hong Kong, Shatin, Hong Kong; P J Maddison, Gwynedd Rheumatology Service, Ysbyty Gwynedd, Bangor, Wales, UK; Y A Mekori, Department of Medicine, Meir Hospital, Kfar Saba, Israel; T Miyamae, Department of Paediatrics, Yokohama City University School of Medicine, Yokohama, Japan; J Moore, Department of Haematology, St Vincents Hospital, Sydney, Australia; $H M$ Moutsopoulos, Department of Pathophysiology, Medical School, National University of Athens, Athens, Greece; F J Munoz-Rodriguez, Department of Autoimmune Diseases, Hospital Clinic, Barcelona, Catalonia, Spain; J Musial, Jagiellonian University School of Medicine, Krakow, Poland; A Nakajima, Institute of Rheumatology, Tokyo Women's Medical University, Tokyo, Japan; M C Neuwelt, Medical Service, VA Palo Alto Health Care System, California, USA; A Parke, Department of Internal Medicine, Division of Rheumatic Diseases, University of Connecticut Health Center, Connecticut, USA; S Praprotnik, Univerisity Clinical Centre, Department of Rheumatology, Ljubljana, Slovenia; $B$ Roca, Department of Internal Medicine, Hospital General de Castelló, Castelló, Spain; J Rojas-Rodriguez, Department of Rheumatology, Specialties Hospital, Manuel Avila Camacho National Medical Centre, Puebla, Mexico; $R$ Roldan, Rheumatology Department, Hospital Reina Sofia, Cordoba, Spain; A D Sawitzke, Division of Rheumatology, Department of Internal Medicine, University of Utah School of Medicine, Salt Lake City, Utah, USA; C G Schaar, Department of Haematology, Leiden University Medical Centre, Leiden, Netherlands; A Šipek-Dolnicar, Department of Rheumatology,
University Medical Center, Ljubljana, Slovenia; $A$ C Spyropoulos, Clinical Thrombosis Center, Albuquerque, New Mexico, USA; $R$ Sinico, Nephrology and Dialysis Unit and Centre of Clinical Immunology and Rheumatology, San Carlo Borromeo Hospital, Milan, Italy; L Stojanovich, ClinicalHospital Centre "Bezhanijska Kosa", Belgrade, Yugoslavia; $M$ Tektonidou, Department of Pathophysiology, Medical School, National University of Athens, Athens, Greece; $C$ Vasconcelos, Hospital General de San Antonio, Porto, Portugal; M Wislowska, Outpatients Department of Rheumatology, Central Clinical Hospital, Warsaw, Poland.

\section{REFERENCES}

1 Levi $M$, de Jonge $E$, van der Poll T, ten Cate H. Advances in the understanding of the pathogenetic pathways of disseminated intravascular coagulation result in more insight in the clinical picture and better management strategies. Semin Thromb Hemost 2001; 27:569-75.

2 Levi $M$, ten Cate H. Disseminated intravascular coagulation. N Engl J Med 1999;341:586-92.

3 Muller-Berghaus G, ten Cate $H$, Levi M. Disseminated intravascular coagulation: clinical spectrum and established as well as new diagnostic approaches. Thromb Haemost 1999;82:706-12.

4 Asherson RA. The catastrophic antiphospholipid syndrome. J Rheumatol 1992;19:508-12.

5 Cervera R, Piette J-C, Font J, Khamashta MA, Shoenfeld Y, Camps MT, et al. Antiphospholipid syndrome: clinical and immunologic manifestations and patterns of disease expression in a cohort of 1,000 patients. Arthritis Rheum 2002;46:1019-27.

6 Asherson RA, Cervera R, de Groot PG, Erkan D, Boffa M-C, Piette J-C, et al. Catastrophic antiphospholipid syndrome: international consensus statement on classification criteria and treatment guidelines. Lupus 2003; 12:530-4.

7 Asherson RA, Cervera R, Piette J-C, Font J, Lie JT, Burcoglu A, et al. Catastrophic antiphospholipid syndrome: clinical and laboratory features of 50 patients. Medicine (Baltimore) 1998;77:195-207.

8 Asherson RA, Cervera R, Piette J-C, Shoenfeld Y, Espinosa G, Petri MA, et al. Catastrophic antiphospholipid syndrome. Clues to the pathogenesis from a series of 80 patients. Medicine (Baltimore) 2001;80:355-77.

9 Cervera R, Gomez-Puerta JA, Espinosa G, Cucho M, Font J. "CAPS registry": a review of 200 cases from the international registry of patients with catastrophic antiphospholipid syndrome (CAPS). Ann Rheum Dis 2003;62(suppl 1):88.

10 Taylor FB, Toh CH, Hoots WK, Wada H, Levi M. Towards definition, clinical and laboratory criteria, and a scoring system for disseminated intravascular coagulation. Thromb Haemost $2001 ; 86: 1327-30$.

11 Triplett DA, Asherson RA. Pathophysiology of the catastrophic antiphospholipid syndrome (CAPS). Am J Hematol 2000;65:154-9.

12 De Groot PG, Horbach DA, Derksen RHWM. Protein C and other cofactors involved in the binding of antiphospholipid antibodies: relation to the pathogenesis of thrombosis. Lupus 1996;5:488-93.

13 Tassies D, Espinosa G, Munoz-Rodriguez FJ, Freire C, Cervera R, Monteagudo J, et al. The $4 \mathrm{G} / 5 \mathrm{G}$ polymorphism of the type 1 plasminogen activator inhibitor gene and thrombosis in patients with antiphospholipid syndrome. Arthritis Rheum 2000;43:2349-58.

14 McKay DG. Diseases of hypersensitivity: disseminated intravascular coagulation. Arch Intern Med 1965;116:83-94.

15 Asherson RA, Shoenfeld Y. The role of infection in the pathogenesis of catastrophic antiphospholipid syndrome - molecular mimicry? J Rheumatol 2000;27:12-14. 